PROCEEDINGS OF THE

AMERICAN MATHEMATICAL SOCIETY

Volume 134, Number 1, Pages 167-175

S 0002-9939(05)08279-1

Article electronically published on August 11, 2005

\title{
UPPER ESTIMATES FOR THE ENERGY OF SOLUTIONS OF NONHOMOGENEOUS BOUNDARY VALUE PROBLEMS
}

\author{
ALFONSO CASTRO AND MÓNICA CLAPP
}

(Communicated by Jonathan M. Borwein)

\begin{abstract}
We establish upper bounds for the energy of critical levels of the functional associated to a perturbed superlinear elliptic boundary value problem. We show that the perturbed problem satisfies the estimates obtained by Bahri and Lions (1988) for the symmetric problem. We use these estimates to prove the existence of nonradial solutions to a radial elliptic boundary value problem. Our results fill a gap in an earlier paper by Aduén and Castro.
\end{abstract}

\section{Introduction AND STATEMENT OF RESUlts}

Let $\Omega$ be a bounded smooth domain in $\mathbb{R}^{N}$ with $N \geq 3$, and let $H \equiv H_{0}^{1}(\Omega)$ be the Sobolev space of square integrable functions in $\Omega$ having first-order partial derivatives in $L^{2}(\Omega)$. In [3] A. Bahri and P.L. Lions proved that for $p \in\left(2, \frac{2 N-2}{N-2}\right)$ and $f \in C^{0}(\bar{\Omega})$ the elliptic problem

$$
\begin{cases}-\Delta u=|u|^{p-2} u+f & \text { in } \Omega, \\ u=0 & \text { on } \partial \Omega\end{cases}
$$

has a sequence of solutions $\left\{u_{k}\right\}$ which satisfy

$$
J\left(u_{k}\right) \geq C_{1} k^{\gamma}
$$

for some positive constant $C_{1}$ and $\gamma=\frac{2 p}{N(p-2)}$, where $J: H \rightarrow \mathbb{R}$ is the functional defined by

$$
J(u)=\frac{1}{2} \int_{\Omega}|\nabla u|^{2}-\frac{1}{p} \int_{\Omega}|u|^{p}-\int_{\Omega} f u .
$$

The proofs in [3] are based on ideas developed in [2] combined with the semiclassical inequality due to M. Cwickel [6], E.H. Lieb [10] and Rosenbljum [11. Actually, in [2] the existence of the $u_{k}$ 's was established for further restricted values of $p$. The arguments in [2] and [3] rely on the analysis of the symmetric case $f=0$ for which $J$ has a sequence $\left(c_{k}\right)$ of critical values which satisfy

$$
C_{2} k^{\gamma} \leq c_{k} \leq C_{3} k^{\gamma}
$$

If the boundary condition in $(\wp)$ is replaced by $u=u_{0}$, with $u_{0} \in C^{2}(\bar{\Omega})$ and $\Delta u_{0}=0$, the existence of an unbounded sequence of solutions $\left(u_{k}\right)$ was established

Received by the editors April 20, 2004.

2000 Mathematics Subject Classification. Primary 35J20, 58E05; Secondary 34B15.

Key words and phrases. Critical points, Morse index, nonradial solutions, semiclassical inequality, perturbed nonlinear elliptic equation.

This research was partially supported by PAPIIT, UNAM, México, under grant IN110902-3. 
by P. Bolle, N. Ghoussoub and H. Tehrani in [5] under the additional restriction $p<\frac{2 N}{N-1}$. In this case problem $(\wp)$ is equivalent to problem

$$
\begin{cases}-\Delta u=\left|u+u_{0}\right|^{p-2}\left(u+u_{0}\right)+f & \text { in } \Omega, \\ u=0 & \text { on } \partial \Omega,\end{cases}
$$

and the solutions of $\left(\wp^{\prime}\right)$ are the critical points of the functional $J: H \rightarrow \mathbb{R}$ given by

$$
J(u)=\frac{1}{2} \int_{\Omega}|\nabla u|^{2}-\frac{1}{p} \int_{\Omega}\left|u+u_{0}\right|^{p}-\int_{\Omega} f u .
$$

Here we prove that (1.1) also holds for the nonsymmetric problem $\left(\wp^{\prime}\right)$. Namely, we prove the following.

Theorem 1.1. If $u_{0}=0$ and $p<\frac{2 N-2}{N-2}$, or if $u_{0} \neq 0$ and $p<\frac{2 N}{N-1}$, then $J$ has an unbounded sequence of critical values $\left(\widetilde{c}_{k}\right)$ which satisfy

$$
\widetilde{c}_{k} \leq C k^{\gamma}
$$

where $C$ is a positive constant and $\gamma=2 p / N(p-2)$.

Theorem 1.1 fills a gap in the proof of Theorem 3 in 1 where (1.2) was used. As an application of the existence of exactly two radial solutions of problem $\left(\wp^{\prime}\right)$ with $k$ nodal domains for large enough $k$ (see proof of Theorem 3 in 1]) we obtain the following.

Theorem 1.2. If $\Omega$ is a ball or an annulus, $u_{0}$ is a constant function, $f=0$, and $p<\frac{2 N}{N-1}$, then problem $\left(\wp^{\prime}\right)$ has infinitely many nonradial solutions.

The reader is refered to 8,9 , and references therein, for recent results on the existence of nonsymmetric solutions for symmetric problems.

The proof of Theorem 1.1 requires some precise knowledge of the topology of the sublevel sets of the functional

$$
I(u)=\frac{1}{2} \int_{\Omega}|\nabla u|^{2}-\frac{1}{p} \int_{\Omega}|u|^{p}
$$

associated to the symmetric problem. Let

$$
I^{c}=\{u \in H: I(u) \leq c\}
$$

and let $\pi_{k}\left(I^{c}, I^{0} \backslash\{0\}\right)$ be the $k$-th relative homotopy group (or homotopy set if $k=1$ ) with any base point in $I^{0} \backslash\{0\}$ (for the definition of these groups see for example [12, Chapter 7]). We shall prove the following.

Theorem 1.3. There exist $\alpha, \beta>0$, depending only on $\Omega$ and $p$, such that for every $c \geq 0$ the homomorphism induced by the inclusion

$$
\pi_{k}\left(I^{c}, I^{0} \backslash\{0\}\right) \rightarrow \pi_{k}\left(I^{\alpha c+\beta}, I^{0} \backslash\{0\}\right)
$$

is trivial for all $k \geq 1$.

In other words, given a map $\varphi: \mathbb{B}^{k} \rightarrow I^{c}$ such that $\varphi\left(\mathbb{S}^{k-1}\right) \subset I^{0} \backslash\{0\}$, there exists a homotopy $\Theta: \mathbb{B}^{k} \times[0,1] \rightarrow I^{\alpha c+\beta}$ such that $\Theta(x, 0)=\varphi(x)$ for all $x \in \mathbb{B}^{k}$ and $\Theta\left(\mathbb{S}^{k-1} \times[0,1] \cup \mathbb{B}^{k} \times\{1\}\right) \subset I^{0} \backslash\{0\}$.

Here $\mathbb{B}^{k}=\left\{x \in \mathbb{R}^{k}:|x| \leq 1\right\}$ is the unit ball and $\mathbb{S}^{k-1}=\left\{x \in \mathbb{R}^{k}:|x|=1\right\}$ is the unit sphere in euclidean $k$-space $\mathbb{R}^{k}$.

This paper is organized as follows. In section 2 we derive Theorem 1.1 from Theorem 1.3 and apply it to prove Theorem 1.2. Section 3 is devoted to the proof of Theorem 1.3 


\section{CRitical Values of PERTURBed SymmetriC FunCtionals}

We start by recalling a critical point result due to Bolle, Ghoussoub and Tehrani.

Let $X$ be an infinite-dimensional Hilbert space and let $\Phi: X \times[0,1] \rightarrow \mathbb{R}$ be a $C^{2}$-functional. We think of $\Phi$ as being a path of functionals

$$
\Phi_{t}: X \rightarrow \mathbb{R}, \quad \Phi_{t}(u)=\Phi(u, t), \quad 0 \leq t \leq 1,
$$

and denote by $\Phi_{t}^{\prime}(u)=\frac{\partial}{\partial u} \Phi(u, t)$ the derivative of $\Phi_{t}$. Assume that $\Phi$ has the following properties.

(P1) Every sequence $\left(u_{n}, t_{n}\right) \in X \times[0,1]$ such that $\left(\Phi_{t_{n}}\left(u_{n}\right)\right)$ is bounded and $\left\|\Phi_{t_{n}}^{\prime}\left(u_{n}\right)\right\| \rightarrow 0$ has a convergent subsequence.

(P2) For every $b \in \mathbb{R}$ there is a constant $C$ such that

$$
\left|\frac{\partial}{\partial t} \Phi(u, t)\right| \leq C\left(\left\|\Phi_{t}^{\prime}(u)\right\|+1\right)(\|u\|+1) \quad \text { if }\left|\Phi_{t}(u)\right| \leq b .
$$

(P3) There exist two continuous functions $\theta_{1}, \theta_{2}:[0,1] \times \mathbb{R} \rightarrow \mathbb{R}, \theta_{1} \leq \theta_{2}$, which are Lipschitz continuous on the second variable and such that

$$
\theta_{1}\left(t, \Phi_{t}(u)\right) \leq \frac{\partial}{\partial t} \Phi(u, t) \leq \theta_{2}\left(t, \Phi_{t}(u)\right) \quad \text { if } \Phi_{t}^{\prime}(u)=0 .
$$

(P4) $\Phi_{0}$ is even and, for every finite-dimensional subspace $W$ of $X$,

$$
\sup _{0 \leq t \leq 1} \Phi_{t}(w) \rightarrow-\infty \quad \text { as } w \in W,\|w\| \rightarrow \infty
$$

Fix a sequence of linear subspaces $X_{1} \subset \cdots \subset X_{k} \subset \cdots$ of $X$ with $\operatorname{dim} X_{k}=k$, and define

$$
c_{k}=\inf _{\varphi \in \Gamma} \sup _{x \in X_{k}} \Phi_{0}(\varphi(x))
$$

where

$\Gamma=\{\varphi \in C(X, X): \varphi$ is odd and $\exists R>0$ such that $\varphi(x)=x$ for $\|x\|>R\}$.

Let $\zeta_{i}:[0,1] \times \mathbb{R} \rightarrow \mathbb{R}, i=1,2$, be defined by

$$
\left\{\begin{array}{l}
\zeta_{i}(0, s)=s \\
\frac{\partial}{\partial t} \zeta_{i}(t, s)=\theta_{i}\left(t, \zeta_{i}(t, s)\right)
\end{array}\right.
$$

The following result was proved in [5] (see Proof of Theorem 2.2).

Theorem 2.1 (Bolle, Ghoussoub, Tehrani). Assume that $\Phi$ satisfies (P1)-(P4). If $\zeta_{2}\left(1, c_{k}+\varepsilon\right)<\zeta_{1}\left(1, c_{k+1}\right)$ for some $\varepsilon>0$, then for every $\varphi \in \Gamma$ such that $\sup _{\varphi\left(X_{k}\right)} \Phi_{0}<c_{k}+\varepsilon$ there is a critical value $\widetilde{c}_{k}$ of $\Phi_{1}$ which satisfies

$$
\zeta_{2}\left(1, c_{k}\right)<\zeta_{1}\left(1, c_{k+1}\right) \leq \widetilde{c}_{k} \leq \zeta_{2}\left(1, \sup _{x \in X_{k+1}} \Phi_{0}(\varphi(x))\right)
$$

Moreover, if the sequence

$$
\left(\frac{c_{k+1}-c_{k}}{\max _{0 \leq t \leq 1}\left|\theta_{1}\left(t, c_{k+1}\right)\right|+\max _{0 \leq t \leq 1}\left|\theta_{2}\left(t, c_{k}\right)\right|+1}\right)
$$

is unbounded, then $\left(\widetilde{c}_{k}\right)$ is unbounded. 
We shall apply this result to the path of functionals

$$
I_{t}(u)=\frac{1}{2} \int_{\Omega}|\nabla u|^{2}-\frac{1}{p} \int_{\Omega}\left|u+t u_{0}\right|^{p}-t \int_{\Omega} f u, \quad u \in H_{0}^{1}(\Omega) .
$$

Note that $I_{0}=I$ and $I_{1}=J$. We write

$$
\|u\|=\left(\int_{\Omega}|\nabla u|^{2}\right)^{1 / 2}, \quad|u|_{p}=\left(\int_{\Omega}|u|^{p}\right)^{1 / p}
$$

for the norm in the Sobolev space $H_{0}^{1}(\Omega)$ of square integrable functions in $\Omega$ having first-order partial derivatives in $L^{2}(\Omega)$, and the norm in $L^{p}(\Omega)$ respectively. The following corollary of Theorem 1.3 will be proved in the following section.

Corollary 2.2. There are constants $\alpha, \beta>0$, depending only on $\Omega$ and $p$, with the following property: For every pair of finite-dimensional subspaces $V \subset W$ of $H_{0}^{1}(\Omega)$ with $\operatorname{dim} W=\operatorname{dim} V+1$, every odd $\operatorname{map} \varphi: V \rightarrow H_{0}^{1}(\Omega)$ and every $R>0$ such that $\varphi(v)=v$ if $\|v\| \geq R$, there is an $\widetilde{R} \geq R$ and an odd map $\widetilde{\varphi}: W \rightarrow H_{0}^{1}(\Omega)$ which satisfies:

(i) $\widetilde{\varphi}(v)=\varphi(v)$ for every $v \in V$,

(ii) $\widetilde{\varphi}(w)=w$ for every $w \in W$ with $\|w\| \geq \widetilde{R}$,

(iii) $\max _{w \in W} I(\widetilde{\varphi}(w)) \leq \alpha \max _{v \in V} I(\varphi(v))+\beta$.

We apply this result to prove Theorem 1.1

Proof of Theorem 1.1. Bolle, Ghoussoub and Tehrani [5] showed that $I_{t}$ satisfies properties (P1)-(P4) with $\theta_{2}(t, s)=A\left(s^{2}+1\right)^{1 / 4}=-\theta_{1}(t, s)$ if $u_{0} \neq 0$. If $u_{0}=0$ it is easy to see that $I_{t}$ satisfies these properties with $\theta_{2}(t, s)=A\left(s^{2}+1\right)^{1 / 2 p}=-\theta_{1}(t, s)$. Let $X_{k} \subset H_{0}^{1}(\Omega)$ be the space spanned by the first $k$ Dirichlet eigenfunctions of $-\Delta$ and let

$$
c_{k}=\inf _{\varphi \in \Gamma} \sup _{u \in X_{k}} I(\varphi(u))
$$

where $\Gamma=\left\{\varphi \in C\left(H_{0}^{1}(\Omega), H_{0}^{1}(\Omega)\right): \varphi\right.$ is odd, $\varphi(u)=u$ for $\|u\|$ large enough $\}$. Using the estimate

$$
C_{1} k^{\gamma} \leq c_{k}
$$

with $\gamma=2 p / N(p-2)$ [3, 13, Bolle, Ghoussoub and Tehrani [5] showed that the sequence (2.3) is unbounded, provided $u_{0}=0$ and $p<\frac{2 N-2}{N-2}$, or $u_{0} \neq 0$ and $p<\frac{2 N}{N-1}$. Hence $J$ has an unbounded sequence of critical values in those cases. If $\zeta_{2}\left(1, c_{k}+\varepsilon\right)<\zeta_{1}\left(1, c_{k+1}\right)$ for some $0<\varepsilon<1$, choose $\varphi \in \Gamma$ such that $\sup I\left(\varphi\left(X_{k}\right)\right)<c_{k}+\varepsilon$ and apply Corollary 2.2 to obtain an odd map $\widetilde{\varphi}: X_{k+1} \rightarrow$ $H_{0}^{1}(\Omega)$ such that $\widetilde{\varphi}(u)=\varphi(u)$ for $u \in X_{k}, \widetilde{\varphi}(u)=u$ for $\|u\|>R$, and

$$
\sup I\left(\widetilde{\varphi}\left(X_{k+1}\right)\right) \leq \alpha\left(c_{k}+\varepsilon\right)+\beta<\alpha c_{k}+\delta .
$$

By Tietze's extension theorem $\widetilde{\varphi}$ can be extended to an odd map $\widetilde{\varphi}: H_{0}^{1}(\Omega) \rightarrow$ $H_{0}^{1}(\Omega)$ which satisfies $\widetilde{\varphi}(u)=u$ for $\|u\|>R$. It follows from inequality (2.2) in Theorem 2.1 that $J$ has a critical value $\widetilde{c}_{k}$ which satisfies

$$
\widetilde{c}_{k} \leq \zeta_{2}\left(1, \sup I\left(\widetilde{\varphi}\left(X_{k+1}\right)\right)\right) \leq \zeta_{2}\left(1, \alpha c_{k}+\delta\right) .
$$

By definition of $\zeta_{2}$ (see (2.1)),

$$
\left|s-\zeta_{2}(t, s)\right| \leq C_{1}\left|\theta_{2}(s)\right| \leq C_{2}\left(s^{2}+1\right)^{1 / 4} .
$$


On the other hand, Bahri and Lions [3] showed that

$$
c_{k} \leq C_{2} k^{\gamma}
$$

with $\gamma=2 p / N(p-2)$. It follows that

$$
\widetilde{c}_{k} \leq C k^{\gamma}
$$

for some positive constant $C$.

Remark 2.3. Note that, since $\zeta_{2}(t, c)$ is nondecreasing in $t$, inequalities (2.2) and (2.4) immediately imply that

$$
C^{\prime} k^{\gamma} \leq \widetilde{c}_{k}
$$

for some positive constant $C^{\prime}$.

Proof of Theorem 1.2. From the proof of Theorem 3 in [1] it follows that problem $\left(\wp^{\prime}\right)$ has at most two radial solutions with $k$ interior nodal hypersurfaces, for each $k$ large enough. Theorem 1.1 above, together with Theorem 1 in [1] (or Theorem 4 in [7]), imply that $\left(\wp^{\prime}\right)$ has infinitely many nonradial solutions.

\section{EXTENSIONS OF ODD MAPS WITH LINEAR ENERGY ESTIMATES}

The purpose of this section is to prove Theorem 1.3 We split the proof into several lemmas.

Lemma 3.1. Let $\Omega$ be a bounded smooth domain in $\mathbb{R}^{N}$. Then there exists $a \in \mathbb{R}$ with the following properties:

(i) There exists $x^{\prime} \in \mathbb{R}^{N-1}$ such that $\left(x^{\prime}, a\right) \in \Omega$.

(ii) If $\left(x^{\prime}, b\right) \in \Omega$ and $b \geq a$, then $\left(x^{\prime}, t\right) \in \Omega$ for all $a \leq t \leq b$.

Proof. Let $e_{N}=(0, \ldots, 0,1) \in \mathbb{R}^{N}$, let $M=\max \left\{x \cdot e_{N}: x \in \bar{\Omega}\right\}$ and let $K=\{x \in$ $\left.\bar{\Omega}: x \cdot e_{N}=M\right\}$. Then $K$ is a compact subset of $\partial \Omega$. Let $\nu: \partial \Omega \rightarrow \mathbb{R}^{N}$ be the outer unit normal vector field, and let $\mathcal{O}=\left\{x \in \partial \Omega: \nu(x) \cdot e_{N}>0\right\}$. Then $\mathcal{O}$ is an open neighborhood of $K$ in $\partial \Omega$ and, since $K$ is compact, there is an $a<M$ such that the set $A=\left\{x \in \partial \Omega: x \cdot e_{N} \geq a\right\} \subset \mathcal{O}$. Thus, for every $\left(x^{\prime}, t\right) \in A$ there exists $\varepsilon>0$ such that $\left(x^{\prime}, s\right) \notin \Omega$ if $t<s<t+\varepsilon$ and $\left(x^{\prime}, s\right) \in \Omega$ if $t-\varepsilon<s<t$. It follows that, for every $\left(x^{\prime}, t\right) \in A$,

$$
\left(\left\{x^{\prime}\right\} \times[a, M]\right) \cap \partial \Omega=\left\{\left(x^{\prime}, t\right)\right\} \quad \text { and } \quad\left\{x^{\prime}\right\} \times[a, t) \subset \Omega
$$

as claimed.

Set

$$
I^{\#}(u)=2 \int_{\Omega}|\nabla u|^{2}-\frac{1}{p} \int_{\Omega}|u|^{p} .
$$

Lemma 3.2. There exists an even continuous function $\tau: H_{0}^{1}(\Omega) \rightarrow[0, \infty)$ with the following properties:

(i) $\quad I([(1-s)+s \tau(u)] u) \leq I(u)$ for every $u \in H_{0}^{1}(\Omega), 0 \leq s \leq 1$.

(ii) If $I^{\#}(u) \leq 0$, then $\tau(u)=1$.

(iii) If $2 I(u) \leq \max _{t \geq 0} I(t u)$, then $I^{\#}(\tau(u) u) \leq 0$.

(iv) $I^{\#}(\tau(u) u) \leq \max \{\alpha I(u), 0\}$ with $\alpha=2^{(3 p-2) /(p-2)}$. 
Proof. For every $v \in H_{0}^{1}(\Omega)$ with $\|v\|=1$, define $0<t_{v}^{-}<\widehat{t}_{v}<t_{v}^{+}<T_{v}<\infty$ as follows:

$$
\begin{aligned}
I\left(\widehat{t_{v}} v\right) & =\max _{t \geq 0} I(t v), \\
2 I(t v) & \geq \max _{t \geq 0} I(t v) \Longleftrightarrow t \in\left[t_{v}^{-}, t_{v}^{+}\right], \\
2\left(T_{v}\right)^{2} & =\frac{1}{p}|v|_{p}^{p}\left(T_{v}\right)^{p} .
\end{aligned}
$$

For $t \geq 0$ set

$$
\rho(t v)= \begin{cases}0 & \text { if } 0 \leq t \leq t_{v}^{-}, \\ \widehat{t}_{v}\left(t-t_{v}^{-}\right) /\left(\widehat{t}_{v}-t_{v}^{-}\right) & \text {if } t_{v}^{-} \leq t \leq \widehat{t}_{v}, \\ \left(T_{v}-\widehat{t}_{v}\right)\left(t-\widehat{t}_{v}\right) /\left(t_{v}^{+}-\widehat{t}_{v}\right)+\widehat{t}_{v} & \text { if } \widehat{t}_{v} \leq t \leq t_{v}^{+}, \\ T_{v} & \text { if } t_{v}^{+} \leq t \leq T_{v}, \\ t & \text { if } T_{v} \leq t\end{cases}
$$

and, for $u=t v \in H_{0}^{1}(\Omega)$ with $\|v\|=1, t \geq 0$, set

$$
\tau(u)=\frac{\rho(t v)}{t} .
$$

Then $\rho(t v) \leq t$ if $0 \leq t \leq \widehat{t}_{v}$, and $\rho(t v) \geq t$ if $\widehat{t}_{v} \leq t$. Therefore (i) holds. Property (ii) follows immediately from the definition. If $2 I(t v) \leq \max _{s \geq 0} I(s u)=I\left(\widehat{t}_{v} v\right)$, then either $0 \leq t \leq t_{v}^{-}$or $t_{v}^{+} \leq t$. In the first case $\rho(t v)=0$. In the second case $\rho(t v) \geq T_{v}$. Hence, in both cases,

$$
2\|\tau(u) u\|^{2}=2\|\rho(t v) v\|^{2} \leq \frac{1}{p}|\rho(t v) v|_{p}^{p}=\frac{1}{p}|\tau(u) u|_{p}^{p} .
$$

This proves (iii). Finally, it is easy to see that

$$
\max _{t \geq 0} I^{\#}(t u)=2^{2 p /(p-2)} \max _{t \geq 0} I(t u) .
$$

Hence

$$
I^{\#}(\tau(u) u) \leq 2^{(3 p-2) /(p-2)} I(u) \quad \text { if } \max _{t \geq 0} I(t u) \leq 2 I(u) .
$$

This, combined with (iii), yields (iv).

For any subspace $V$ of $H_{0}^{1}(\Omega)$ and any $R>0$ we write

$$
B_{R} V=\{v \in V:\|v\| \leq R\}, \quad S_{R} V=\{v \in V:\|v\|=R\} .
$$

Lemma 3.3. Let $V$ be a finite-dimensional subspace of $H_{0}^{1}(\Omega)$ and let $R>0$ be such that $I(v) \leq 0$ for those $v \in V$ with $\|v\| \geq R$. Then, for every map $\varphi: B_{R} V \rightarrow I^{c}$ such that $c \geq 0$ and $\varphi\left(S_{R} V\right) \subset I^{0} \backslash\{0\}$, there exists a homotopy $\Theta: B_{R} V \times[0,1] \rightarrow$ $I^{c}$ such that

(i) $\Theta(v, 0)=\varphi(v)$ for every $v \in B_{R} V$,

(ii) $\Theta(z, 1)=z$ for every $z \in S_{R} V$,

(iii) $\Theta\left(S_{R} V \times[0,1]\right) \subset I^{0} \backslash\{0\}$.

Proof. Since $I^{0} \backslash\{0\}$ is homotopy equivalent to the unit sphere in $H_{0}^{1}(\Omega)$, it is contractible. Hence there is a homotopy $\Psi: S_{R} V \times[0,1] \rightarrow I^{0} \backslash\{0\}$ with $\Psi(z, 0)=$ $\varphi(z)$ and $\Psi(z, 1)=z$. Define $\vartheta: B_{R+1} V \rightarrow I^{c}$ as follows:

$$
\vartheta(v)= \begin{cases}\varphi(v) & \text { if } \quad\|v\| \leq R \\ \Psi\left(R \frac{v}{\|v\|},\|v\|-R\right) & \text { if } R \leq\|v\| \leq R+1 .\end{cases}
$$


Now define $\Theta: B_{R} V \times[0,1] \rightarrow I^{c}$ by

$$
\Theta(v, t)=\vartheta\left(\frac{R+t}{R} v\right) .
$$

It is easy to see that $\Theta$ has the desired properties.

Lemma 3.4. There exist $\alpha, \beta>0$, depending only on $\Omega$ and $p$, with the following property: Given a finite-dimensional subspace $V$ of $H_{0}^{1}(\Omega)$, a map $\varphi: V \rightarrow H_{0}^{1}(\Omega)$ and an $R>0$ such that $\varphi(v)=v$ if $\|v\| \geq R$, there exist a map $\psi: V \times[0, \infty) \rightarrow$ $H_{0}^{1}(\Omega)$ and an $R^{\prime} \geq R$ which satisfy:

(i) $\psi(v, 0)=\varphi(v)$ for every $v \in V$,

(ii) $\psi(v, r) \in I^{0} \backslash\{0\} \quad$ if $\|v\| \geq R^{\prime}$ or $r \geq R^{\prime}$,

(iii) $I(\psi(v, r)) \leq \alpha \max \{I(\varphi(v)), 0\}+\beta$ for every $v \in V, r \geq 0$.

Proof. Let $a$ be as in Lemma 3.1. We may assume without loss of generality that $a=$ 0 . Let $\tau: H_{0}^{1}(\Omega) \rightarrow \mathbb{R}$ be as in Lemma 3.2, We write $x=\left(x^{\prime}, x_{N}\right) \in \mathbb{R}^{N-1} \times \mathbb{R} \equiv \mathbb{R}^{N}$. Given $u \in H_{0}^{1}(\Omega)$ and $0 \leq s \leq 2$ define

$$
u_{s}(x)= \begin{cases}{[(1-s)+s \tau(u)] u(x)} & \text { if } 0 \leq s \leq 1, \\ \tau(u) u\left(x^{\prime}, s x_{N}\right) & \text { if } x_{N} \geq 0,1 \leq s \leq 2, \\ \tau(u) u\left(x^{\prime}, x_{N}\right) & \text { if } x_{N} \leq 0,1 \leq s \leq 2 .\end{cases}
$$

Here $u\left(x^{\prime}, t\right)=0$ if $\left(x^{\prime}, t\right) \notin \Omega$. Then $u_{s} \in H_{0}^{1}(\Omega)$ for every $0 \leq s \leq 2$, and

$$
\int_{\Omega}\left|\nabla u_{s}\right|^{2} \leq s \int_{\Omega}|\nabla(\tau(u) u)|^{2} \quad \text { and } \quad \int_{\Omega}\left|u_{s}\right|^{p} \geq s^{-1} \int_{\Omega}|\tau(u) u|^{p} \quad \text { if } 1 \leq s \leq 2 .
$$

Hence

$$
s I\left(u_{s}\right) \leq \frac{s^{2}}{2} \int_{\Omega}|\nabla(\tau(u) u)|^{2}-\frac{1}{p} \int_{\Omega}|\tau(u) u|^{p} \quad \text { if } 1 \leq s \leq 2 .
$$

This inequality and Lemma 3.2 yield

$$
I\left(u_{s}\right) \leq \begin{cases}I(u) & \text { if } 0 \leq s \leq 1, \\ \max \left\{I^{\#}(\tau(u) u), 0\right\} & \text { if } 1 \leq s \leq 2 .\end{cases}
$$

Let $\Omega_{2}=\left\{\left(x^{\prime}, \frac{1}{2} x_{N}\right): x \in \Omega, x_{N} \geq 0\right\} \cup\left\{x \in \Omega: x_{N} \leq 0\right\}$. Since $\Omega_{2} \varsubsetneqq \Omega$ because of Lemma 3.1. we may choose $\omega \in C_{c}^{\infty}\left(\Omega \backslash \Omega_{2}\right), \omega \neq 0$, with

$$
\int_{\Omega}|\nabla \omega|^{2}=\int_{\Omega}|\omega|^{p}
$$

Given a map $\varphi: V \rightarrow H_{0}^{1}(\Omega)$ and an $R>0$ such that $\varphi(v)=v$ if $\|v\| \geq R$, define $\psi: V \times[0, \infty) \rightarrow H_{0}^{1}(\Omega)$ by

$$
\psi(v, r)= \begin{cases}\varphi(v)_{r}, & v \in V, 0 \leq r \leq 2 \\ \varphi(v)_{2}+(r-2) \omega, & v \in V, 2 \leq r\end{cases}
$$

Then $\psi$ is an extension of $\varphi$, and (3.1) yields

$$
I(\psi(v, r)) \leq \begin{cases}I(\varphi(v)) & \text { if } 0 \leq r \leq 1 \\ \max \left\{I^{\#}(\tau(\varphi(v)) \varphi(v)), 0\right\} & \text { if } 1 \leq r \leq 2, \\ \max \left\{I^{\#}(\tau(\varphi(v)) \varphi(v)), 0\right\}+I((r-2) \omega) & \text { if } 2 \leq r .\end{cases}
$$

The third inequality follows because $\varphi(v)_{2}$ and $\omega$ have disjoint supports. Hence, Lemma 3.2 yields

$$
I(\psi(v, r)) \leq \alpha \max \{I(\varphi(v)), 0\}+\beta \quad \text { for every } v \in V, r \geq 0,
$$


with $\alpha=2^{(3 p-2) /(p-2)}$ and $\beta=I(\omega)$. Finally, let $R^{\prime}>R$ be such that

$$
\begin{cases}I(v) \leq 0, \quad \tau(v)=1, \quad \text { and } \quad I^{\#}(v) \leq-I(\omega) & \text { if } v \in V, \quad\|v\| \geq R^{\prime}, \\ I((r-2) \omega) \leq-\max _{v \in V} I^{\#}(\tau(\varphi(v)) \varphi(v)) & \text { if } r \geq R^{\prime} .\end{cases}
$$

Since $\varphi(v)=v$ if $\|v\| \geq R^{\prime} \geq R$, and since $I(t \omega) \leq I(\omega)$ for every $t \geq 0$ by (3.2), it follows that

$$
I(\psi(v, r)) \leq 0 \quad \text { if } \quad\|v\| \geq R^{\prime} \text { or } r \geq R^{\prime} .
$$

Proof of Theorem 1.3. Let $\sigma: \mathbb{B}^{k} \rightarrow I^{c}$ be a map such that $\sigma\left(\mathbb{S}^{k-1}\right) \subset I^{0} \backslash\{0\}$. Composing this map with a homeomorphism $\vartheta: B_{R} V \cong \mathbb{B}^{k}$ and applying Lemma 3.3. we may assume that $\sigma \circ \vartheta$ is the restriction to $B_{R} V$ of a map $\varphi: V \rightarrow I^{c}$ defined on a $k$-dimensional subspace $V$ of $H_{0}^{1}(\Omega)$ which satisfies $\varphi(v)=v$ if $\|v\| \geq$ $R$. Let $\psi: V \times[0, \infty) \rightarrow H_{0}^{1}(\Omega)$ and an $R^{\prime} \geq R$ be as in Lemma 3.4 Define $\Theta: B_{R} V \times[0,1] \rightarrow H_{0}^{1}(\Omega)$ by

$$
\Theta(v, s)= \begin{cases}\varphi\left(\left[(1-2 s)+2 s \frac{R^{\prime}}{R}\right] v\right) & \text { if } 0 \leq s \leq \frac{1}{2}, \\ \psi\left(\frac{R^{\prime}}{R} v,(2 s-1) R^{\prime}\right) & \text { if } \frac{1}{2} \leq s \leq 1 .\end{cases}
$$

Then $\Theta(v, 0)=\varphi(v)$ for all $v \in B_{R} V, \Theta\left(S_{R} V \times[0,1] \cup B_{R} V \times\{1\}\right) \subset I^{0} \backslash\{0\}$, and

$$
I(\Theta(v, s)) \leq \alpha c+\beta \quad \text { for all }(v, s) \in B_{R} V \times[0,1]
$$

as claimed.

Proof of Corollary 2.2. For the given $\varphi$ and $R$, let $\psi: V \times[0, \infty) \rightarrow H_{0}^{1}(\Omega)$ and $R^{\prime}>R$ be as in Lemma 3.4. Fix $e \in W$ orthogonal to $V$ with $\|e\|=1$, and extend $\varphi$ to the half space $W^{+}=\{v+r e: v \in V, r \geq 0\}$ by

$$
\bar{\varphi}(v+r e)=\psi(v, r) \quad \text { if } v \in V, r \geq 0 .
$$

Then there exists $R^{\prime \prime} \geq R^{\prime}$ such that $\bar{\varphi}(w) \in I^{0} \backslash\{0\}$ for all $w \in W^{+}$with $\|w\|=R^{\prime \prime}$. Since $I^{0} \backslash\{0\}$ is homotopy equivalent to the unit sphere in $H_{0}^{1}(\Omega)$, it is contractible. Hence, there is a homotopy

$$
\Psi:\left\{w \in W^{+}:\|w\|=R^{\prime \prime}\right\} \times[0,1] \rightarrow I^{0} \backslash\{0\}
$$

such that $\Psi(w, 0)=\bar{\varphi}(w), \Psi(w, 1)=w$, and $\Psi(v, t)=v$ for $v \in V, t \in[0,1]$. Let $\widetilde{R}=R^{\prime \prime}+1$ and define

$$
\widetilde{\varphi}(w)= \begin{cases}\bar{\varphi}(w) & \text { if } w \in W^{+},\|w\| \leq R^{\prime \prime}, \\ \frac{\|w\|}{R^{\prime \prime}} \Psi\left(R^{\prime \prime} \frac{w}{\|w\|},\|w\|-R^{\prime \prime}\right) & \text { if } w \in W^{+}, \quad R^{\prime \prime} \leq\|w\| \leq \widetilde{R}, \\ w & \text { if } w \in W^{+}, \widetilde{R} \leq\|w\|, \\ -\widetilde{\varphi}(-w) & \text { if }-w \in W^{+} .\end{cases}
$$

Since $\varphi$ is odd, $\widetilde{\varphi}$ is well-defined and it is, by definition, an odd extension of $\varphi$ to $W$ which satisfies $\widetilde{\varphi}(w)=w$ if $\|w\| \geq \widetilde{R}$. Note that $r u \in I^{0} \backslash\{0\}$ if $u \in I^{0} \backslash\{0\}$ and $|r| \geq 1$. Hence $I(\widetilde{\varphi}(w)) \leq 0$ if $\|w\| \geq R^{\prime \prime}$ and, by Lemma 3.4.

$$
I(\widetilde{\varphi}(v+r e))=I(\psi(v,|r|)) \leq \alpha \max \{I(\varphi(v)), 0\}+\beta \quad \text { if }\|v+r e\| \leq R^{\prime \prime},
$$

as claimed. 


\section{REFERENCES}

[1] H. Aduén and A. Castro, Infinitely many nonradial solutions to a superlinear Dirichlet problem, Proc. of the Am. Math. Soc. 131 (2002), 835-843. MR1937421 (2003m:35056)

[2] A. Bahri and H. Berestycki, A perturbation method in critical point theory and applications, Trans. Amer. Math. Soc. 267 (1981), 1-32. MR0621969 (82j:35059)

[3] A. Bahri and P.L. Lions, Morse index of some min-max critical points. I. Application to multiplicity results, Comm. Pure Appl. Math. 41 (1988),1027-1037. MR0968487 (90b:58035)

[4] Ph. Bolle, On the Bolza problem, J. Diff. Eq. 152 (1999), 274-288. MR.1674537 (99m:58043)

[5] Ph. Bolle, N. Ghoussoub and H. Tehrani, The multiplicity of solutions to non-homogeneous boundary value problems, Manuscripta Math. 101 (2000), 325-350. MR1751037|(2001c:58010)

[6] M. Cwickel, Weak type estimates for singular values and the number of bound states of Schrödinger operators, Ann. Math. 106 (1977), 93-102. MR0473576 (57:13242)

[7] R. Kajikiya, Radially symmetric solutions of semilinear elliptic equations, existence and Sobolev estimates, Hiroshima Math. J. 21 (1991), 111-161. MR.1091434 (92a:35061)

[8] R. Kajikiya, Orthogonal group invariant solutions of the Emden-Fowler equation, Nonlinear Analysis TMA 44 (2001), 845-896. MR1827891(2003a:35063)

[9] R. Kajikiya, Non-radial solutions with orthogonal subgroup invariance for semilinear Dirichlet problems, Topol. Meth. Nonl. Anal. 21 (2003), 41-51. MR1980135 (2004a:35072)

[10] E. H. Lieb, Bounds on the eigenvalues of the Laplace and Schrödinger operators, Bull. Amer. Math. Soc. 82 (1976), 751-753. MR0407909 (53:11679)

[11] G. Rosenbljum, The distribution of the discrete spectrum for singular differential operators, Soviet Math. Dokl. 13 (1972), 245-249.

[12] E.H. Spanier, Algebraic topology, McGraw-Hill, New York 1966. MR0210112 (35:1007)

[13] K. Tanaka, Morse indices at critical points related to the symmetric mountain pass theorem and applications, Commun. in Partial Diff. Eq. 14 (1989), 99-128. MR0973271 (90i:58019)

Department of Mathematics, Harvey Mudd College, Claremont, California 91711

E-mail address: castro@math.hmc.edu

Instituto de Matemáticas, Universidad Nacional Autónoma de México, Circuito Exterior, Ciudad Universitaria, 04510 México, D.F., México

E-mail address: mclapp@math.unam.mx 